\title{
CANONICAL FORMS OF CERTAIN VOLTERRA INTEGRAL OPERATORS AND A METHOD OF SOLVING THE COMMUTATOR EQUATIONS WHICH INVOLVE THEM ${ }^{1}$
}

\author{
BY STANLEY J. OSHER
}

Communicated by Louis Nirenberg, January 9, 1967

The similarity properties of Volterra operators on $L_{p}[0,1]$ having reasonably smooth kernels seem to depend entirely on the behavior of the kernel as regards zeros and singularities on the diagonal $x=y$.

If $T_{G}$ is a Volterra operator on $L_{p}[0,1]$, then a study of its similarity properties seems to reduce to the following procedure involving the complex kernel $G(x, y)$.

(1) Classify $G(x, x)$ according to its zeros and singularities on the interval $0 \leqq x \leqq 1$.

(2) Show that $T_{G}$ is similar to a unique $T_{p}$ for $T_{p}$ a canonical kernel of the class of which $G(x, y)$ belongs.

See [1], [2], and [4] for $G(x, y)$ of order $\alpha>0$ i.e.

$$
G(x, y)=(x-y)^{\alpha-1} H(x, y) / \Gamma(\alpha)
$$

with $H(x, x)>0$ and $H(x, y)$ having certain smoothness properties. The canonical form in this case is $K J^{\alpha}$ for

$$
K=\left[\int_{0}^{1}[H(t, t)]^{1 / \alpha} d t\right]^{\alpha}
$$

and

$$
J^{\alpha} f=\int_{0}^{x}\left((x-y)^{\alpha-1} / \Gamma(\alpha)\right) f(y) d y .
$$

See [5] and [6] for $G(x, y)$ of rank 1, i.e.

$$
\begin{aligned}
G(x, x)<0 & \text { if } 0 \leqq x<x_{0}, \\
G(x, x)>0 & \text { if } x_{0}<x \leqq 1, \\
G\left(x_{0}, x_{0}\right)=0 . &
\end{aligned}
$$

The canonical form in this case is $k Q_{a, \nu}$ for unique real $k, a$, and $\nu$ satisfying $0 \leqq a, \nu \leqq 1,0<k$

${ }^{1}$ This research was supported in part by an N.S.F. Cooperative Fellowship at New York University from 10/64 to $6 / 66$ and in part by the U. S. Atomic Energy Commission at Brookhaven National Laboratory. 


$$
\begin{gathered}
\int_{0}^{1} G(t, t) d t=k \int_{0}^{1}(x-a) d x=k / 2(1-2 a) \\
\int_{x_{0}}^{1} G(t, t) d t=k \int_{a}^{1}(x-a) d x=k / 2(1-a)^{2} \\
G_{x}\left(x_{0}, x_{0}\right) /\left(G_{x}\left(x_{0}, x_{0}\right)+G_{y}\left(x_{0}, x_{0}\right)\right)=\nu
\end{gathered}
$$

and

$$
Q_{a, v} f=\int_{0}^{x}(x-a)^{\nu}(t-a)^{1-\nu} f(t) d t
$$

These canonical forms are unique in a sense made precise in [4], [5], [7] and [8].

The important and delicate part of the previous work involved solving an equation of the form

$$
Q_{a, \nu} T_{\Gamma(B)}-T_{\Gamma(B)} Q_{a, \nu}=T_{B}
$$

for the kernel $\Gamma(B)$.

This is equivalent to an integral equation

(2) $\int_{y}^{x}\left[(x-a)^{\nu}(t-a)^{1-\nu} \Gamma(t, y)-\Gamma(x, t)(t-a)^{\nu}(y-a)^{1-\nu}\right] d t$

$$
=B(x, y) \text {. }
$$

Dupras, in his doctoral thesis [1], solved the commutator equation

$$
J^{\alpha} * \Gamma^{(\alpha)}(B)-\Gamma^{(\alpha)}(B) * J^{\alpha}=B \quad \text { for all } \alpha>0
$$

using a certain contour integral. He obtained the result

(4) $\Gamma^{(\alpha)}(B)=\int_{0}^{x-y} b(\sigma, y) d \sigma-(1 / \alpha) \int_{0}^{y} b(x-y, t) d t+R_{\alpha}(x, y)$

for

$$
B(x, y)=\int_{0}^{x-y}\left((x-y-\sigma)^{\alpha} / \Gamma(\alpha+1)\right) b(\sigma, y) d \sigma,
$$

(6) $\quad R_{(\alpha)}(x, y)$ is a certain contour integral depending on $\alpha$.

Consider the Volterra integral operator

$$
Q_{[\alpha, \boldsymbol{a}, \nu, p]}=Q_{\left[\alpha ; a_{1}, a_{2}, \cdots, a_{n} ; \nu_{1}, \cdots, \nu_{n} ; p_{1}, \cdots, p_{n}\right]}
$$

which has the kernel $F(x, y)$ such that

$$
F(x, y)=\left((x-y)^{\alpha-1} / \Gamma(\alpha)\right) G(x, y)
$$


with

$$
\begin{aligned}
G(x, x) & =\prod_{i=1}^{n}\left(x-a_{i}\right)^{p_{i}}, \quad 0 \leqq a_{1}<a_{2} \cdots<a_{n} \leqq 1 \\
G_{x}(x, x) & =\left(\prod_{j=1}^{n}\left(x-a_{j}\right)^{p_{i}}\right)\left(\sum_{i=1}^{n} \nu_{i} /\left(x-a_{i}\right)\right) .
\end{aligned}
$$

We shall later restrict $\alpha, \nu_{j}$ and $p_{j}$ in such a way that $Q[\alpha, \boldsymbol{a}, \boldsymbol{v}, \boldsymbol{p}]$ is always a Volterra integral operator.

Suppose for some complex $k$, we could get

$$
k M_{(1 / l)} S_{R} J^{\alpha} S_{S} M_{l}=Q[\alpha, \boldsymbol{a}, \boldsymbol{v}, \boldsymbol{p}]
$$

for $S_{S}=f(S(x))$ and $M_{l}=l(x) f(x)$ both bounded linear invertible transformations on $L_{p}[0,1]$ with $S^{-1}(x)=R(x)$ or

$$
k u^{-1} J^{\alpha} u=Q[\alpha, \boldsymbol{a}, \boldsymbol{v}, \boldsymbol{p}] .
$$

It would then follow that the solution to the commutator equation

$$
\left[Q_{[\alpha, a, \nu, p]}, T_{X}^{[\alpha, a, \nu, p]}\right]=T_{A}
$$

is

$$
T_{X}^{[\alpha, a, \nu, p]}=u^{-1} T_{\mathrm{r}}(\alpha)\left(u T_{A} u^{-1}\right) u / k
$$

or for brevity

$$
X^{[\alpha, a, \nu, p]}=u^{-1} \Gamma^{(\alpha)}\left(u A u^{-1}\right) u / k .
$$

In reality, the transformations $u$ and $u^{-1}$ will, in general, not be bounded, or for that matter well defined. However, we shall use this formalism to obtain candidates for $X^{[\alpha, a, \nu, p]}$. In addition, the formalism yields a precise definition of $Q_{[\alpha, a, \nu, p]}$.

The author used this method in [6] and obtained the canonical form for operators of rank one, i.e. the $Q_{a, \nu}=Q_{[1 ; a ; \nu ; 1]}$.

The candidate for $X^{[1 ; a ; p ; 1]}$ was found and was used to obtain the real commutator solution. See [5] and [6] for an exhaustive description of the similarity properties of operators of rank one.

It is known that a Volterra operator $T_{H}$ with a reasonably smooth kernel $H(x, y)$ commutes with $J^{\alpha}$ iff $H(x, y)=f(x-y)$.

Thus we would think

$$
\left[T_{N}, Q_{[\alpha, a, \nu, p]}\right]=0
$$

if

$$
T_{N}(x, y)=u^{-1} T_{f(x-y)} u \text {. }
$$


We may use the operators which commute with $Q_{[\alpha, a, v, p]}$ to help make $X^{[\alpha, a, v, p]}$ well defined, i.e.

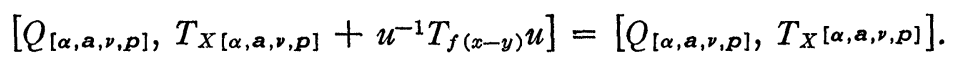

Now we shall ortain the formal expressions for $k, S, l$, and $S^{-1}=R$.

$$
k(R(x)-R(y))^{\alpha-1} R^{\prime}(y)(l(y) / l(x))=(x-y)^{\alpha-1} G(x, y),
$$

$$
\begin{aligned}
k\left[R^{\prime}(x)\right]^{\alpha} & =G(x, x)=\prod_{i=1}^{n}\left(x-a_{i}\right)^{p_{i}} \\
R(x) & =\left(\int_{0}^{x} \prod_{i=1}^{n}\left(t-a_{i}\right)^{p_{i} / \alpha} d t\right) / k^{1 / \alpha}
\end{aligned}
$$

with

$$
k=\left[\int_{0}^{1} \prod_{i=1}^{n}\left(t-a_{i}\right)^{p_{i} / \alpha} d t\right]^{\alpha} \quad \text { so that } R(1)=1 .
$$

(It is possible that $k=0$. We ignore this difficulty and proceed formally.)

If we equate $x$ derivatives at $y=x$, we obtain

$$
\begin{aligned}
l(x)= & \prod_{i=1}^{n}\left(x-a_{i}\right)^{(1 / 2-1 / 2 \alpha) p_{i}-\nu_{i}}, \\
G(x, y)= & \left(\left(\int_{y}^{x} \prod_{i=1}^{n}\left(t-a_{i}\right)^{p_{i} / \alpha} d t\right) /(x-y)\right)^{\alpha-1} \\
& \cdot \prod_{i=1}^{n}\left(x-a_{i}\right)^{\nu_{i}+p_{i}(1 / 2 \alpha-1 / 2)}\left(y-a_{i}\right)^{-\nu_{i}+p_{i}(1 / 2 \alpha+1 / 2)} .
\end{aligned}
$$

In order that $T_{F}$ be a Volterra operator, we require

$$
\alpha \geqq 1, \quad p_{i}(1 / 2-1 / 2 \alpha) \leqq \nu_{i} \leqq p_{i}(1 / 2 \alpha+1 / 2), \quad 0<p_{i} .
$$

(This is not the most general case, but is sufficiently general for our purposes here.)

The corresponding commuting operator should be $T_{N}$ with

$$
\begin{aligned}
N(x, y)= & f(R(x)-R(y)) R^{\prime}(y) l(y) / l(x), \\
N(x, y)= & \prod_{i=1}^{n}\left(x-a_{i}\right)^{v_{i}+p_{i}(1 / 2 \alpha-1 / 2)}\left(y-a_{i}\right)^{p_{i}(1 / 2 \alpha+1 / 2)-v_{i}} \\
& \cdot f\left(\int_{y}^{x} \prod_{i=1}^{n}\left(t-a_{i}\right)^{p_{i} / \alpha} d t\right) .
\end{aligned}
$$


It can be shown formally that

$$
Q_{[\alpha, a, \nu, p]} * Q_{[\beta, a, \nu, p]}=Q_{[\alpha+\beta, a, \nu, p]} .
$$

Acknowledgment. The author wishes to thank Professor J. T. Schwartz for his guidance in this work, which is based upon a doctoral dissertation at New York University.

\section{BIBLIOGRAPHY}

1. A. Dupras, Doctoral Thesis, New York University, New York, 1965.

2. J. M. Freeman, Volterra Operators similar to $J: J: f \rightarrow \int_{0}^{x} f(t) d t$, Trans. Amer. Math. Soc. 116 (1965), 181-192.

3. K. O. Friedrichs, Perturbations of continuous spectra, Comm. Pure Appl. Math. 1(1948), 361-406,

4. G. K. Kalisch, On similarity, reducing manifolds, and unitary equivalence of certain volterra operators, Ann. of Math. (2) 66 (1957), 481-494.

5. S. J. Osher, Necessary conditions for similarity of certain Volterra integral operators, Mem. Amer. Math. Soc. (to appear).

6. - Sufficient conditions for similarily of certain Volterra integral operators, Mem. Amer. Math. Soc. (to appear).

7. G. K. Kalisch, On similarity invariants of certain Volterra operators in $L_{p}$, Pacific J. Math. 11 (1961), 247-252.

8. - On isometric equivalence of certain Volterra operators, Proc. Amer. Math. Soc. 12 (1961), 93-98.

BRookHaVEN NATIONAL LABORATORY 